\title{
Host-Guest Prospects of Neodymium and Gadolinium Ultraphosphate Frameworks for Nuclear Waste Storage: Multi-Temperature Topological Analysis of Nanoporous Cages in $\mathrm{RP}_{5} \mathrm{O}_{14}$
}

Alisha J. Cramer ${ }^{a}$ and Jacqueline M. Cole ${ }^{* a, b, c, d}$

a Cavendish Laboratory, Department of Physics, University of Cambridge, J. J. Thomson Avenue, Cambridge, CB3 OHE. UK.

b ISIS Neutron and Muon Source, STFC Rutherford Appleton Laboratory, Harwell Science and Innovation Campus, Didcot, Oxfordshire, OX11 OQX. UK.

c Argonne National Laboratory, 9700 South Cass Avenue, Argonne, IL 60439, USA.

$d$ Department of Chemical Engineering and Biotechnology, University of Cambridge, West Cambridge Site, Philippa Fawcett Drive, Cambridge, CB3 OFS. UK.

* Author to whom correspondence should be addressed at: jmc61@cam.ac.uk

\section{ABSTRACT}

Rare-earth ultraphosphate $\left(\mathrm{RP}_{5} \mathrm{O}_{14}\right)$ framework materials are potential host media for nuclear waste storage, since cages within their nanoporous structures have volumes that match well those of prospective guests such as uranium or plutonium ions. Good volume matches of host cages and guest compounds are nonetheless not the only structural requirement for ensuring viable nuclear waste storage. Host structures also need to be stable enough to withstand the typical environmental conditions of long-term storage of spent nuclear fuel. To this end, the nanoporous cage topologies of neodymium and gadolinium ultraphosphate, $\mathrm{NdP}_{5} \mathrm{O}_{14}$ and $\mathrm{GdP}_{5} \mathrm{O}_{14}$, are investigated as a function of temperature. Topological analysis shows that, while both compounds are essentially isostructural, thus displaying the same type of cage structures, the cage volumes of $\mathrm{NdP}_{5} \mathrm{O}_{14}$ are significantly larger than those of $\mathrm{GdP}_{5} \mathrm{O}_{14}$, with one stark exception. This exception concerns the smallest cage of $\mathrm{NdP}_{5} \mathrm{O}_{14}$, whose 8/4 topology lacks structural cross-linking that would otherwise give it much more strength. This 'squashed' cage appears to owe its origins to the specific nature of crystallogra phic twinning in $\mathrm{NdP}_{5} \mathrm{O}_{14}$, which causes strain that needs alleviating; squashing the $8 / 4$ cage in $\mathrm{NdP}_{5} \mathrm{O}_{14}$ would be the most susceptible option towards this end, since its cage manifests the weakest structural construct. $\mathrm{GdP}_{5} \mathrm{O}_{14}$ succumbs to a gradual contraction of its $8 / 4$ cage only with increasing temperature above $300 \mathrm{~K}$; this is well below its second-order monoclinic-toorthorhombic phase transition at $420-430 \mathrm{~K}$; which is $420 \mathrm{~K}$ for $\mathrm{NdP}_{5} \mathrm{O}_{14}$. Fortunately, the volumes of heavy metal ions that arise from spent nuclear fuels do not match the size requirements of cages with 8/4 topology in $\mathrm{RP}_{5} \mathrm{O}_{14}$ hosts needed for encapsulation; otherwise, radiation leakage of such containment would present a risk. Both $\mathrm{NdP}_{5} \mathrm{O}_{14}$ and $\mathrm{GdP}_{5} \mathrm{O}_{14}$ would therefore seem to offer good prospects as host media for nuclear waste storage.

\section{KEYWORDS}

Rare-earth ultraphosphate; nuclear waste storage; temperature-dependent nanoporous cage topology; crystallographic twinning 


\section{INTRODUCTION}

Nuclear waste containment is an important field of environmental science given the desperate need to find cleaner, safer, and more sustainable ways to store spent nuclear fuel. Host-guest media is one possible option whereby crystalline materials that contain nanoporous framework structures act as hosts for encapsulating radioactive ions and oxides from nuclear waste streams [1]. Rare-earth ultraphosphates were recently identified as possible host media for nuclear waste storage, in a screening study of a wide range of nanoporous framework materials [2]. The screening study determined the volumes of empty cages in 628 phosphate framework structures, to pinpoint potential host materials that could encapsulate environmentally important guests with similar volumes, such as $\mathrm{UO}_{2}$ and $\mathrm{PuO}_{2}$. Cage volume alone is nonetheless not a sufficient criterion to fully assess the prospects of host media for nuclear waste storage applications. This predictive type of materials screening process accordingly needs to be supplemented by detailed experimental studies on host media that have been short-listed by this materials discovery workflow. In particular, the stability of the cages that are prospected for guest encapsulation needs to be assessed as does any structural characteristic that may perturb the host framework structure in a way that risks the leaching of nuclear waste.

To this end, we herein investigate the topological stability of all cage structures in two rareearth ultraphosphates, neodymium and gadolinium ultraphosphate, $\mathrm{NdP}_{5} \mathrm{O}_{14}$ and $\mathrm{GdP}_{5} \mathrm{O}_{14}$. This includes a temperature-dependent profiling of these cages in the range $\mathrm{T}=120-480 \mathrm{~K}$, given that rare-earth ultraphosphate crystal structures, $\mathrm{RP}_{5} \mathrm{O}_{14}(\mathrm{R}=$ lanthanide), are well known to undergo monoclinic-to-orthorhombic second-order phase transitions with transition temperatures rising with increasing lanthanide atomic number, from approximately $390 \mathrm{~K}\left(\mathrm{LaP}_{5} \mathrm{O}_{14}\right)$ to $447 \mathrm{~K}\left(\mathrm{TbP}_{5} \mathrm{O}_{14}\right)$ [3-7]. This temperature range naturally contains the typical environmental conditions of nuclear waste storage.

Understanding how the cage structures within $\mathrm{RP}_{5} \mathrm{O}_{14}$ frameworks may be perturbed as a consequence of the overarching structural transition of the material is thus crucial for ensuring safety from disastrous incidents such as radiation leakage.

Reports of specific transition temperatures can vary widely owing to differences in factors such as crystal fabrication, or the experimental analysis probe used (e.g. X-ray diffraction, neutron diffraction, or optical assessment) [8]. The well-documented ferroelasticity of $\mathrm{RP}_{5} \mathrm{O}_{14}$ below the Curie temperature demonstrates an additional complication [3-5,9,10], particularly in $\mathrm{NdP}_{5} \mathrm{O}_{14}$, where twin boundaries may be introduced, or moved, by applying surprisingly low amounts of localized pressure $\left(14 \pm 3 \mathrm{kNm}^{-2}\right)[3,5]$. Above the Curie temperature, $\mathrm{RP}_{5} \mathrm{O}_{14}$ crystals become paraelastic [4,9]. The tendency of $\mathrm{NdP}_{5} \mathrm{O}_{14}$ toward twinning in the ferroelastic phase has been extensively investigated, and it has been shown that the natural twin boundaries are of two orientations. The more commonly found a twin corresponds to a change in the crystallographic $c$-axis, while the higher-energy $b$-type twin displays changes in the crystallographic $a$-axis $[3,10]$. Even though $\mathrm{GdP}_{5} \mathrm{O}_{14}$ also exhibits a ferroelastic twin boundary on the (100)-plane [9], studies on this compound remain sparse. Accordingly, the effect of crystallographictwinning on the cage structures of these two subject compounds is studied in detail, using single-crystal X-ray diffraction, with particular attention being directed at the pseudo-merihedral twinning below the transition temperature.

\section{MATERIALS AND METHODS}




\subsection{Data collection}

High quality single crystals of $\mathrm{NdP}_{5} \mathrm{O}_{14}$ and $\mathrm{GdP}_{5} \mathrm{O}_{14}$ were grown according to the procedure reported by Danielmeyer and co-workers [11]. In each instance, a suitable single crystal was mounted onto a nylon fibre using an epoxy resin. Samples were placed onto a Rigaku Saturn $724+$ CCD single-crystal X-ray diffractometer and probed from $120 \mathrm{~K}$ to $480 \mathrm{~K}$ using an Oxford Cryosystems CryostreamPlus $\mathrm{N}_{2}$ cooling device. A full dataset suitable for crystal structure determination was collected for each compound at $5 \mathrm{~K}$ or $10 \mathrm{~K}$ heating intervals. The samples were held at each new temperature for a minimum of 15 minutes. The $\mathrm{GdP}_{5} \mathrm{O}_{14}$ sample was also subsequently cooled within the same temperature range using $5 \mathrm{~K}$ or $10 \mathrm{~K}$ increments, whereupon data collections were acquired to investigate potential hysteresis effects. All data were acquired assuming the lowest possible (either monoclinic or triclinic) crystal symmetry so as to ensure that sufficient data were collected at all temperatures. Cell refinement, data collection, and data reduction were carried out using the Rigaku CrystalClear-SM Expert 2.0 software [12]. The absorption correction was implemented using ABSCOR [13]. All structures were solved using direct methods and refined by full-matrix least-squares methods on F2 using SHELXL-97 [14]. Structures at all temperatures were solved with monoclinic symmetry; higher-temperature structures ( $\geq 340 \mathrm{~K}$ ) were also solved with orthorhombic symmetry. Thus, the pattern of the twin fraction (BASF) through the transition could be followed through the monoclinic structural refinement. This dual refinement option also allowed pinpointing of the transition temperature by observing at which temperature the structure solutions changed from a preference for monoclinic to orthorhombic symmetry, as indicated by statistical figures-of-merit, such as the R1 factor, and the refined twinning fraction. A detailed summary of the crystal, data collection and crystal structure refinement details of $\mathrm{NdP}_{5} \mathrm{O}_{14}$ and $\mathrm{Gd}_{\mathrm{p}} 5 \mathrm{O}_{14}$ at each temperature is given in the Crystallographic Information Files that comprise the Supporting Information.

\subsection{Crystallochemical analysis}

The crystallographic topological analysis program package TOPOS15 (version 4.0 Professional) was used to identify cages in the $\mathrm{RP}_{5} \mathrm{O}_{14}$ structures and to determine the respective void spaces within. For a comprehensive crystallochemical analysis, TOPOS utilizes graph theory; void space analysis is accomplished via a two-step process: $i)$ the determination of all cages found within each structure, prior to ii) calculating the void space volume within each cage using Voronoi-Dirichlet polyhedra (VDP). This procedure establishes a basis for the comparison of the cavity volumes in each structure. The workflow that used TOPOS to ascertain void space contained within individual cages of $\mathrm{GdP}_{5} \mathrm{O}_{14}$ and $\mathrm{NdP}_{5} \mathrm{O}_{14}$ is shown in Figure 1. 


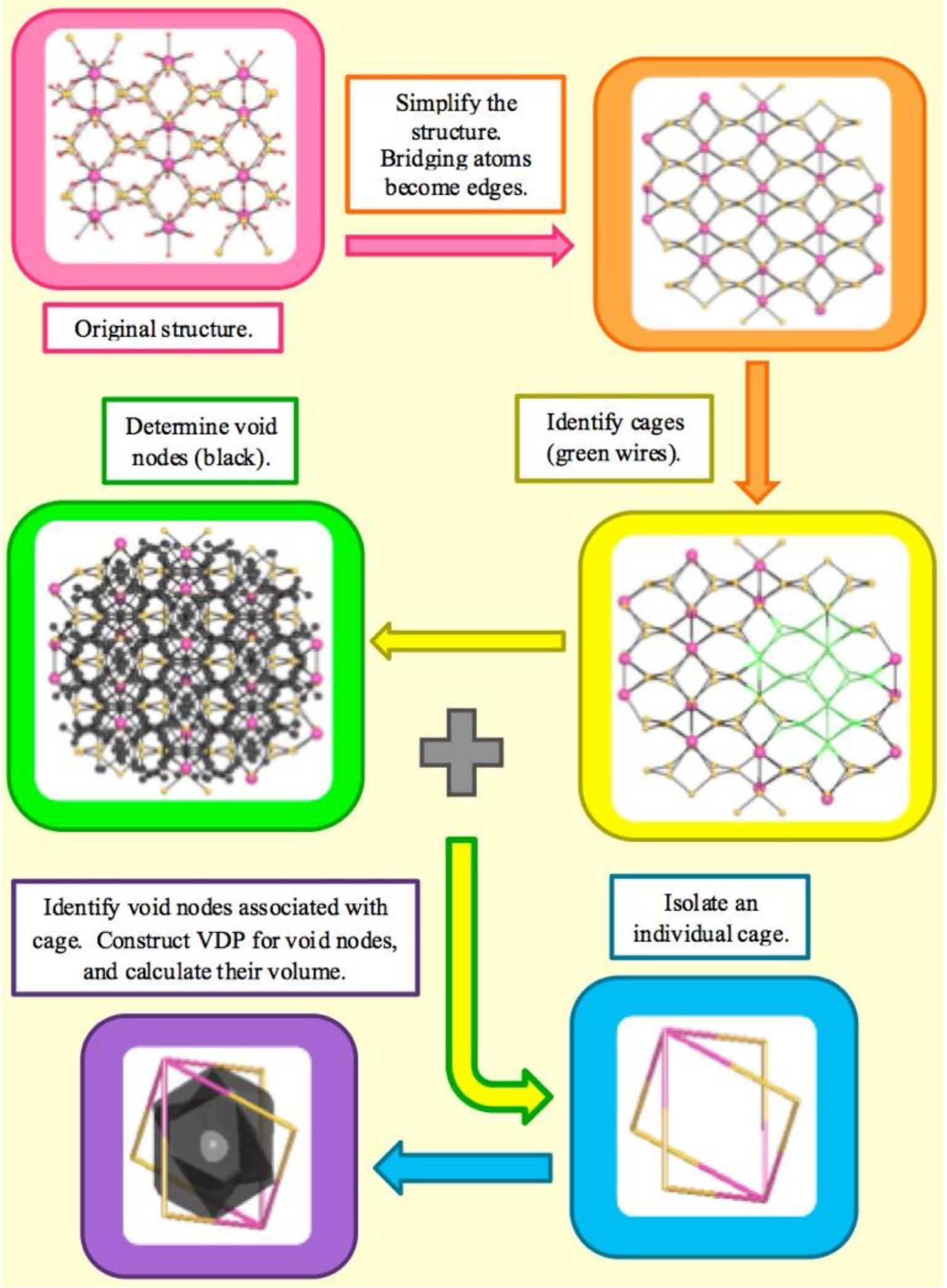

Figure 1. The process of determining void space volumes within cages, using TOPOS.

The framework structures of $\mathrm{GdP}_{5} \mathrm{O}_{14}$ and $\mathrm{NdP}_{5} \mathrm{O}_{14}$ contain tiles, defined as generalized polyhedra (cages), which contain at least two edges incident upon each vertex and two faces incident upon each edge $[16,17]$. Tiles are characterized by how many faces a given tile 
possesses. Each face is defined by its $m$-membered rings, and the tiles are described by face symbols $\left[M^{m} \cdot N^{n} \ldots\right]$, which denominate $m$ faces that are $M$-rings, and $n$ faces that are $N$-rings [16]. The topological representation of nodes and tiles is illustrated in Figure 2 using the example of $\mathrm{NdP}_{5} \mathrm{O}_{14}$. Physically, tiles correspond to minimal cages within a net, from which larger tiles can be constructed by merging the faces of minimal cages, giving rise to maximal proper tiles. For the purposes of this study, 'cages' refer to maximal proper tiles. For an indepth discussion of cages and tiling, see Delgado-Friedrichs and O'Keeffe, and Blatov et al. $[16,18]$. In order to analyze the cavity size of individual cages within $\mathrm{GdP}_{5} \mathrm{O}_{14}$ and $\mathrm{NdP}_{5} \mathrm{O}_{14}$, cages were isolated, and void nodes were generated from the atoms forming the cages. Consequently, VDP were generated for these void nodes, and their volumes were calculated.

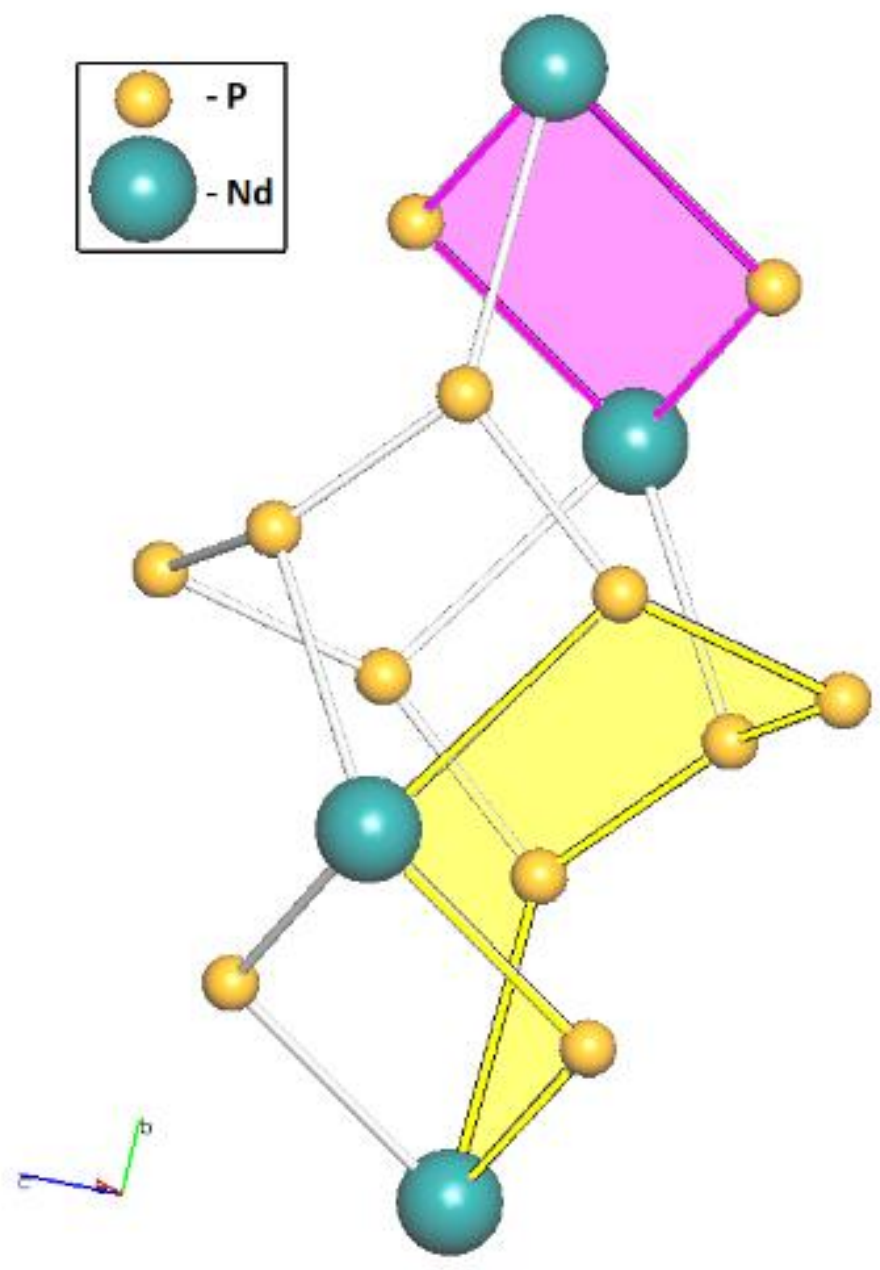

Figure 2. An example of a $16 / 8$ cage using $\mathrm{NdP}_{5} \mathrm{O}_{14}$ defined as a $\left[4^{4} .7^{4}\right]$ tile; $16 / 8$ denominates the total number of nodes/faces; $\left[4^{4} .7^{4}\right]$ indicates the presence of four faces consisting of 4 membered rings (e.g. pink plane), and four faces consisting of 7-membered rings (e.g. yellow plane). Reprinted with permission from ref. [2]. Copyright 2016 American Chemical Society.

\section{RESULTS}

Determination of cage structures in $\mathrm{NdP}_{5} \mathrm{O}_{14}$ and $\mathrm{GdP}_{5} \mathrm{O}_{14}$ below any phase transition. 
The crystal structures of $\mathrm{NdP}_{5} \mathrm{O}_{14}$ and $\mathrm{GdP}_{5} \mathrm{O}_{14}$ at $140 \mathrm{~K}$ are shown in Figure 3.
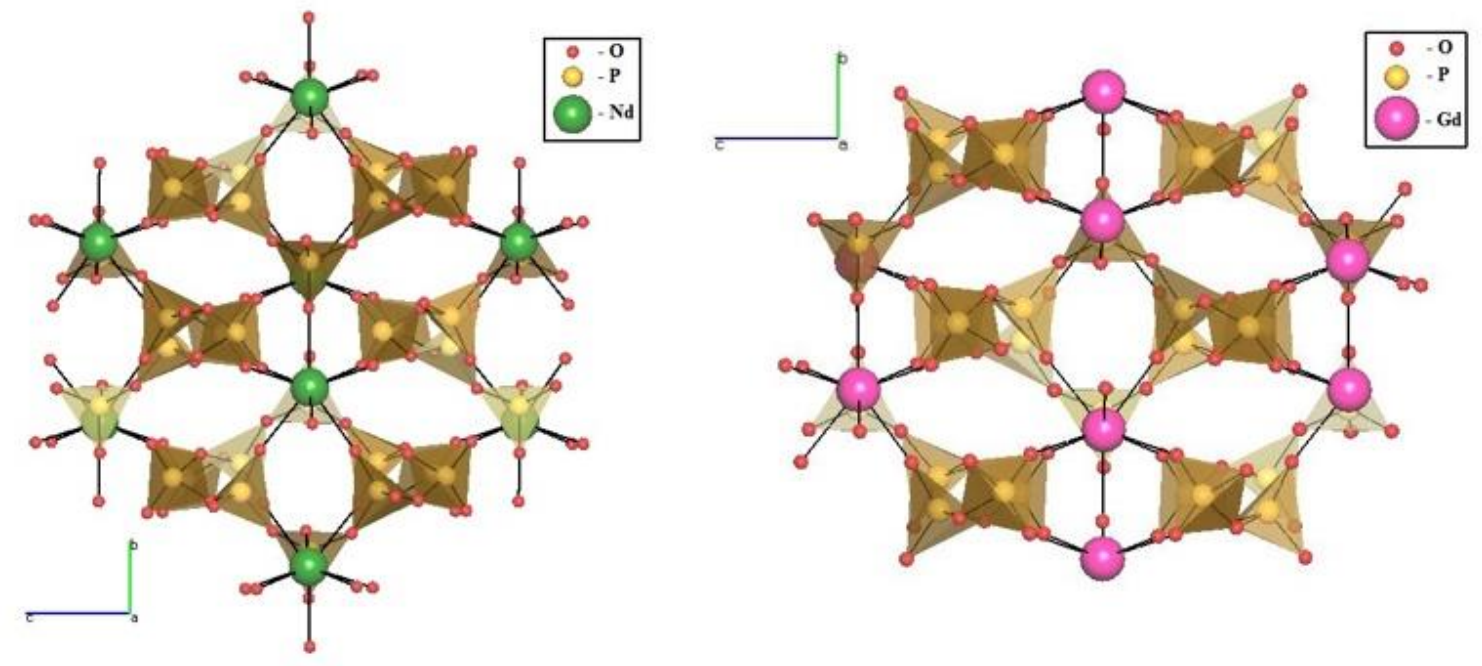

Figure 3. Crystal structures of $\mathrm{NdP}_{5} \mathrm{O}_{14}$ (left), and $\mathrm{GdP}_{5} \mathrm{O}_{14}$ (right), as viewed looking down the (100) crystallographic axis.

A topological analysis of each crystal structure involved an initial determination of the maximal tiles, and an identification of void space volumes for each cage. Both structures exhibit five identified maximal proper tiles: $\left[4^{4}\right], 2\left[4^{2} \cdot 6^{2}\right],\left[4^{2} .5^{4}\right],\left[4^{4} .7^{4}\right]$, and $2\left[4^{4} .5^{2} \cdot 6^{2} .7^{2}\right]$. According to this notation, the first cage, $\left[4^{4}\right]$, consists of four faces that are type-4 rings (where a type $n$-ring has $n$ edges). The second cage, $2\left[4^{2} \cdot 6^{2}\right]$, comprises two faces that are type- 4 rings, and two faces that are type- 6 rings; the ' 2 ' in front of the square brackets denominates the cage ratio, i.e., 1:2:1:1:2. Each cage is more easily described as a ratio of the total number of nodes/faces. This way, the maximal proper tiles are identified as $6 / 4,8 / 4$, $10 / 6,16 / 8$, and $18 / 10$, with respect to the earlier definitions. The shape of each cage is shown in Figures 4-8 together with their cage volume variation as a function of temperature. All data are presented, while data were only fitted up to $440 \mathrm{~K}$ above which there is no longer a smooth trend since the materials have well exceeded their phase transition.

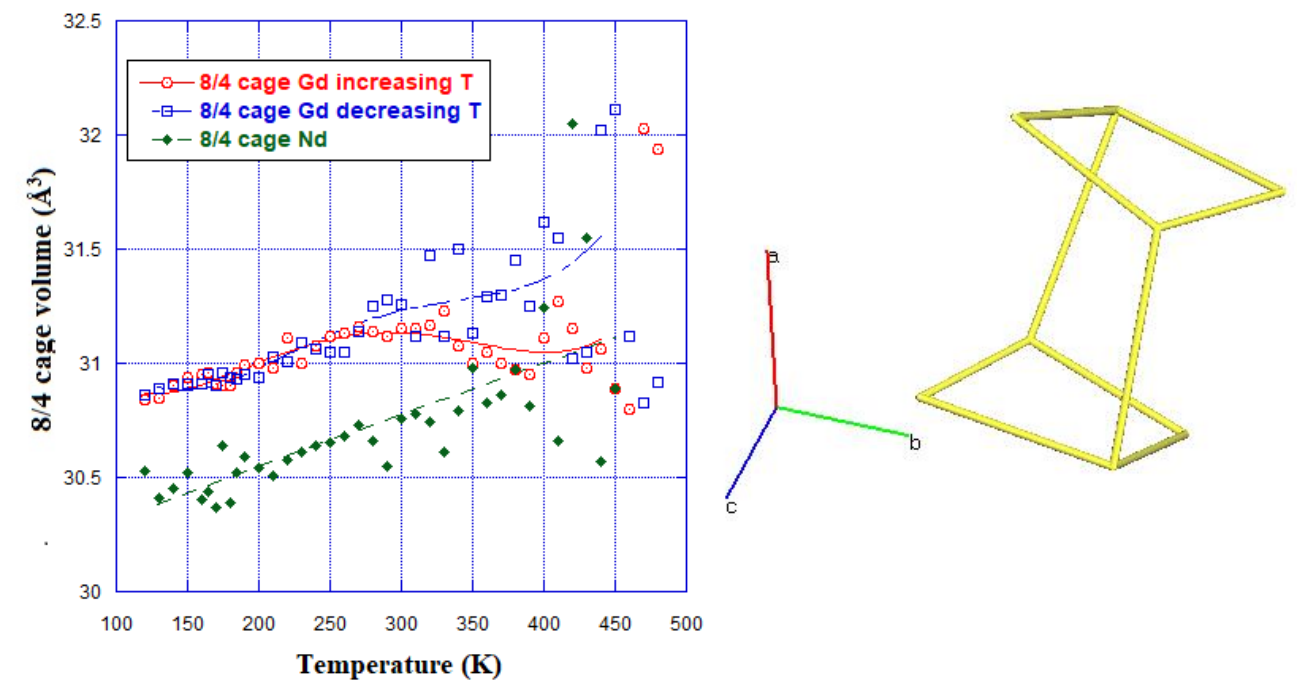


Figure 4. Distribution of the void space volumes within the $8 / 4$ maximal proper tile (left); shape of the $8 / 4$ cage (right).
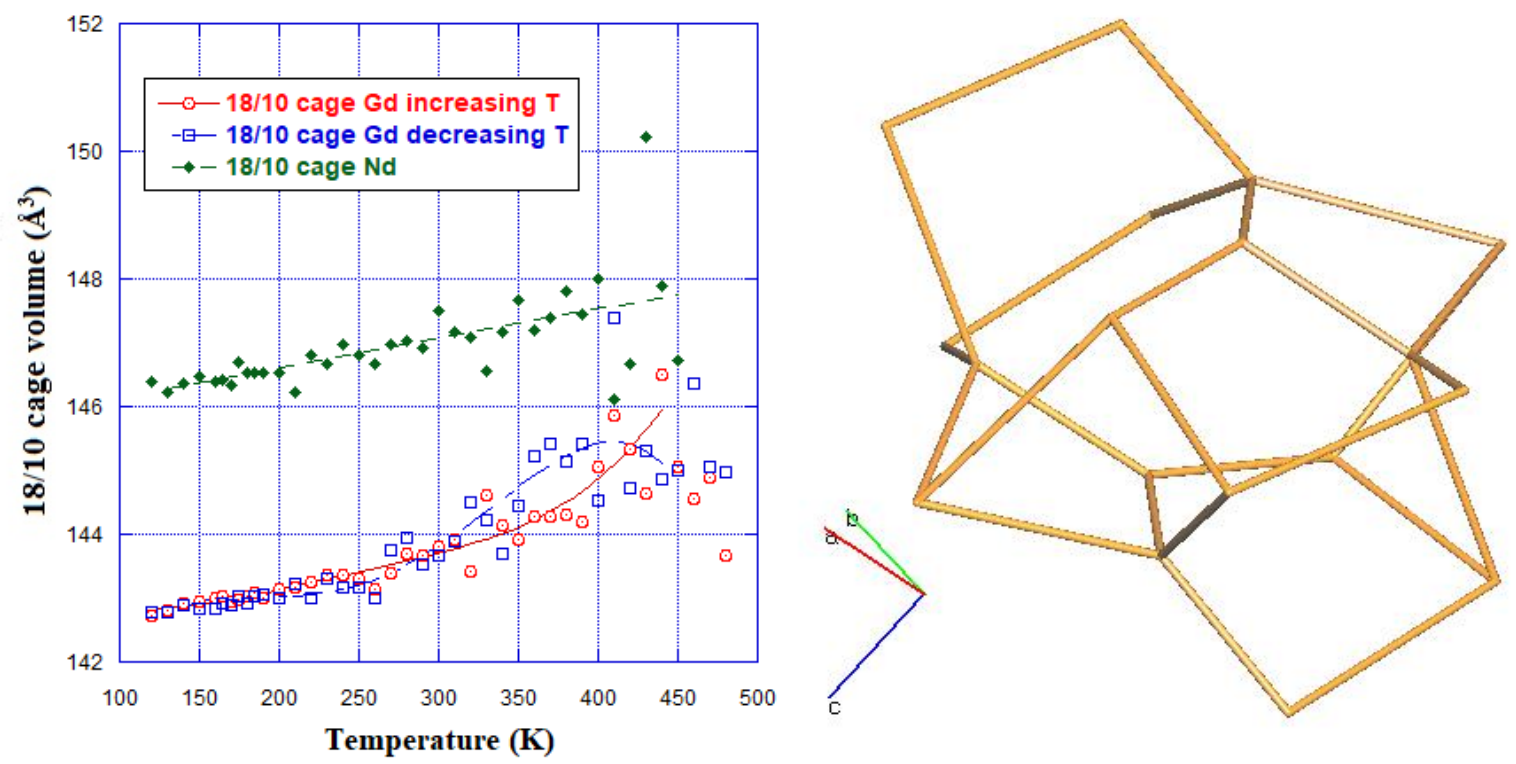

Figure 5. Distribution of the void space volumes within the $8 / 4$ maximal proper tile (left); shape of the $8 / 4$ cage (right).
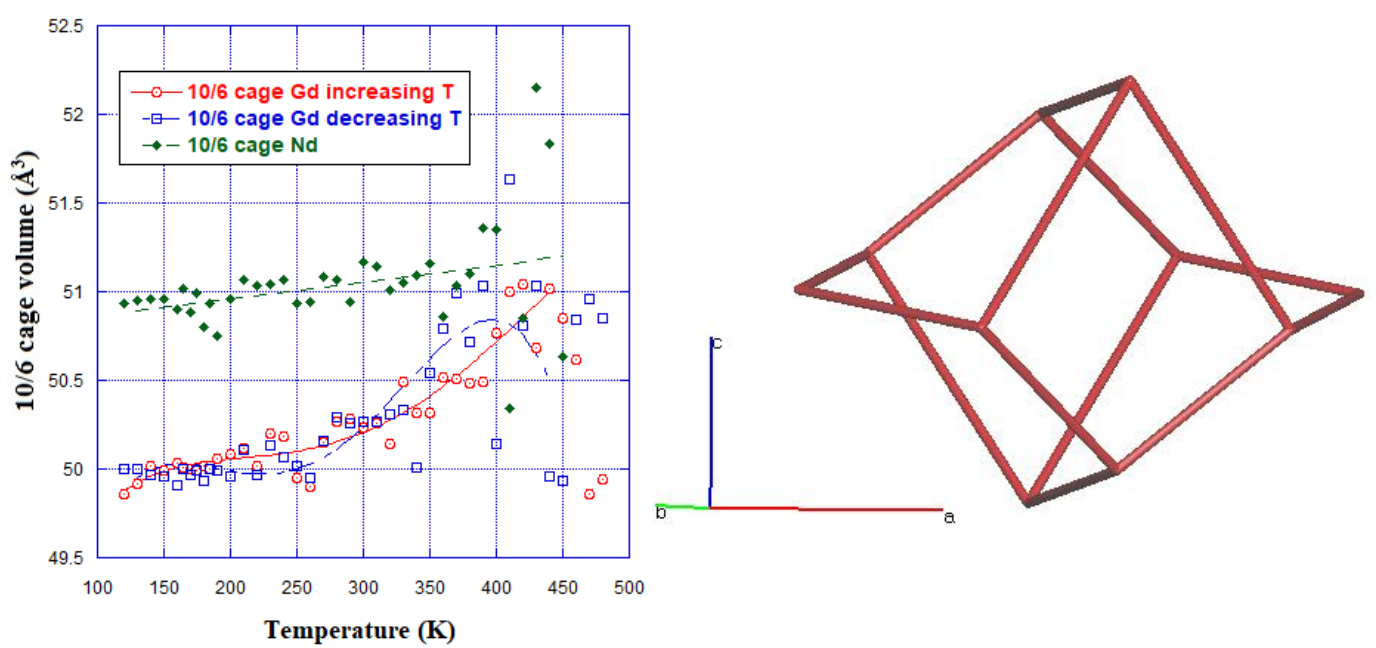

Figure 6. Distribution of the void space volumes within the 10/6 maximal proper tile (left); shape of the $10 / 6$ cage (right). 

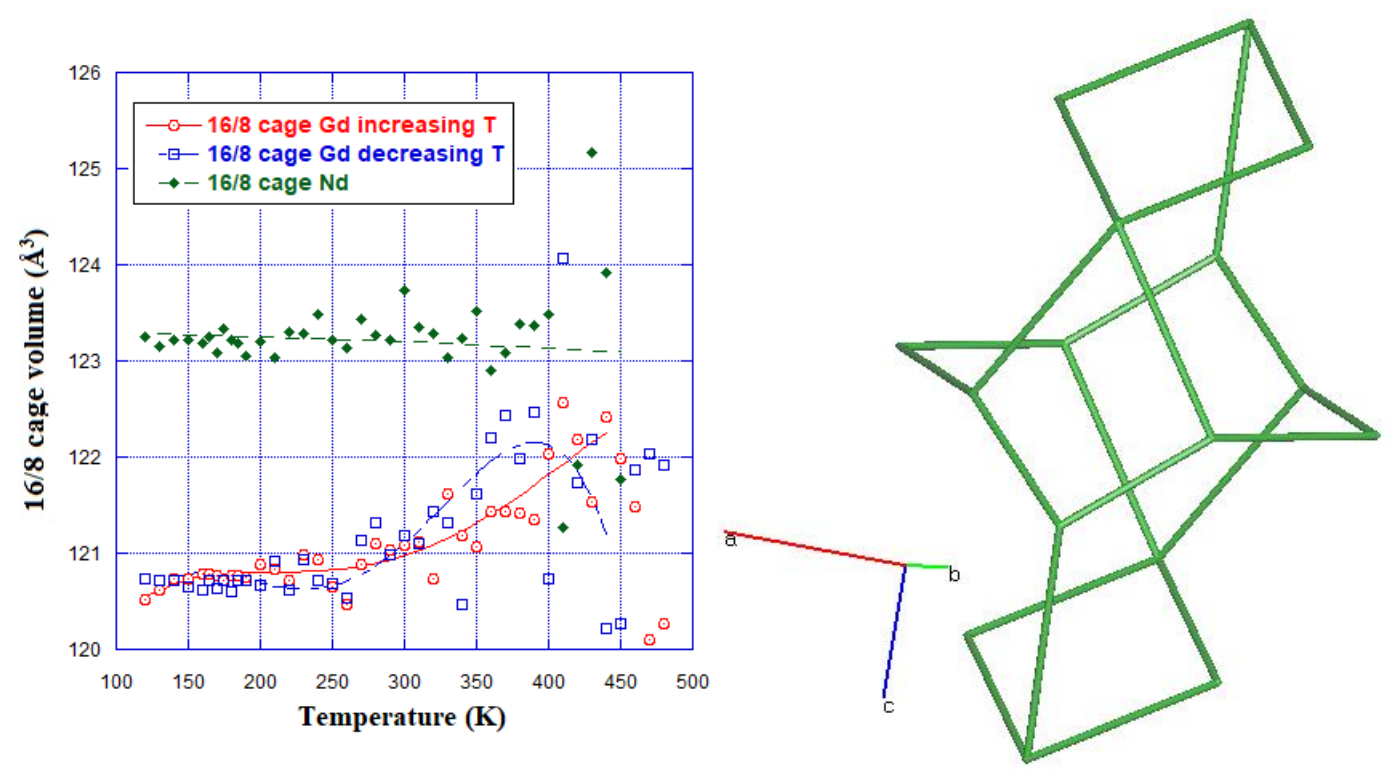

Figure 7. Distribution of the void space volumes within the $16 / 8$ maximal proper tile (left); shape of the $16 / 8$ cage (right).

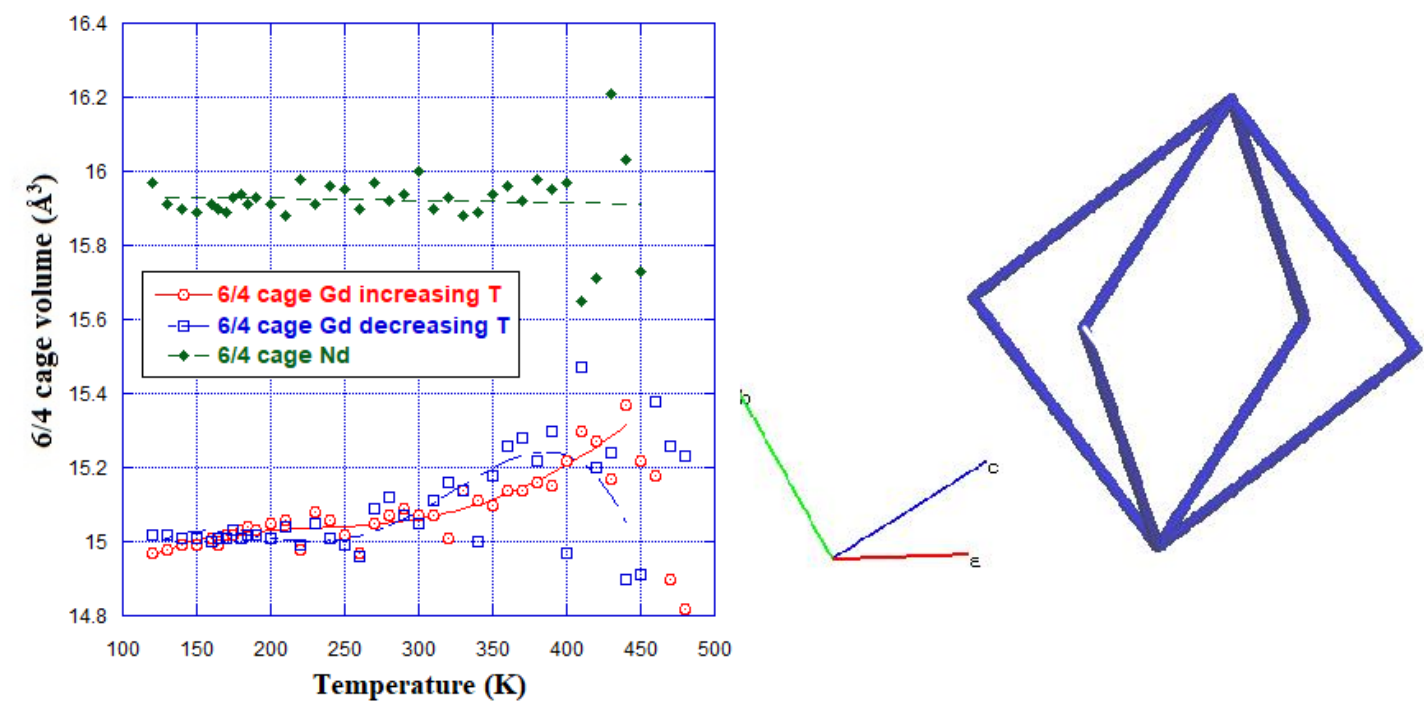

Figure 8. Distribution of the void space volumes within the $6 / 4$ maximal proper tile (left); shape of the 6/4 cage (right).

Trends between cage shape and volume for each compound were first considered using the $120 \mathrm{~K}$ data, i.e. before contemplating any possible perturbations from temperaturedependent effects. For all cages, except for the $8 / 4$ cage, $\mathrm{NdP}_{5} \mathrm{O}_{14}$ produces void space volumes that are $\sim 1-3 \AA^{3}$ larger than those of their $\mathrm{GdP}_{5} \mathrm{O}_{14}$ counterparts. This general trend toward larger cages in $\mathrm{NdP}_{5} \mathrm{O}_{14}$ is perhaps not surprising, as the rare-earth ion coordinates to oxygens, and $\mathrm{R} \cdots \mathrm{O}$ bond lengths in rare-earth ultraphosphate crystal structures [19-24] are slightly longer for $\mathrm{Nd} \cdots \mathrm{O}(2.44 \AA$ ) $)$, than for $\mathrm{Gd} \cdots \mathrm{O}(2.41 \AA$ ) .

The exceptional 8/4 cage is $\sim 0.5 \AA^{3}$ smaller in $\mathrm{NdP}_{5} \mathrm{O}_{14}$ than that in $\mathrm{GdP}_{5} \mathrm{O}_{14}$ (Figure 4). This anomaly in the $8 / 4$ cage void volume of $\mathrm{NdP}_{5} \mathrm{O}_{14}$ is intriguing. From an 'engineering' 
perspective, the $6 / 4,10 / 6,16 / 8$, and $18 / 10$ cages are relatively sturdy, given the good number of supports to hold their framework, while the 8/4 cage is defined by two diamond-shaped 'caps' supported by only two 'columns' (Figure 4, right). In addition, there are no additional nearby physical supports from the framework surrounding the 8/4 cage within the structure, that would otherwise help to stabilize these 'weaker' edges. Since it is the only type of cage that is even smaller than that of its $\mathrm{GdP}_{5} \mathrm{O}_{14}$ counterpart, it would seem that the 8/4 cage in $\mathrm{NdP}_{5} \mathrm{O}_{14}$ may be 'squashed' by some means, owing to its structural weakness relative to all other cages. The observation that the $8 / 4$ cage in $\mathrm{GdP}_{5} \mathrm{O}_{14}$ is not similarly squashed, at least by the same extent, is especially curious, given the structures of $\mathrm{NdP}_{5} \mathrm{O}_{14}$ and $\mathrm{GdP}_{5} \mathrm{O}_{14}$ are essentially isomorphous.

\section{DISCUSSION}

\subsection{Rationalizing the 'squashed' nature of the $8 / 4$ cage in $\mathrm{NdP}_{5} \mathrm{O}_{14}$ by comparing twinning effects in $\mathrm{NdP}_{5} \mathrm{O}_{14}$ and $\mathrm{GdP}_{5} \mathrm{O}_{14}$.}

The positioning of the $8 / 4$ cage within the structure is pertinent, since its 'weak' edge is located at an angle between the crystallographic $b$-and $c$-axes. Given that the predominant twinning within $\mathrm{NdP}_{5} \mathrm{O}_{14}$ crystals is found as a reversal of the c-axis, this consequently puts strain on this cage in such a way as to potentially shorten that particular edge (see Figure 9).

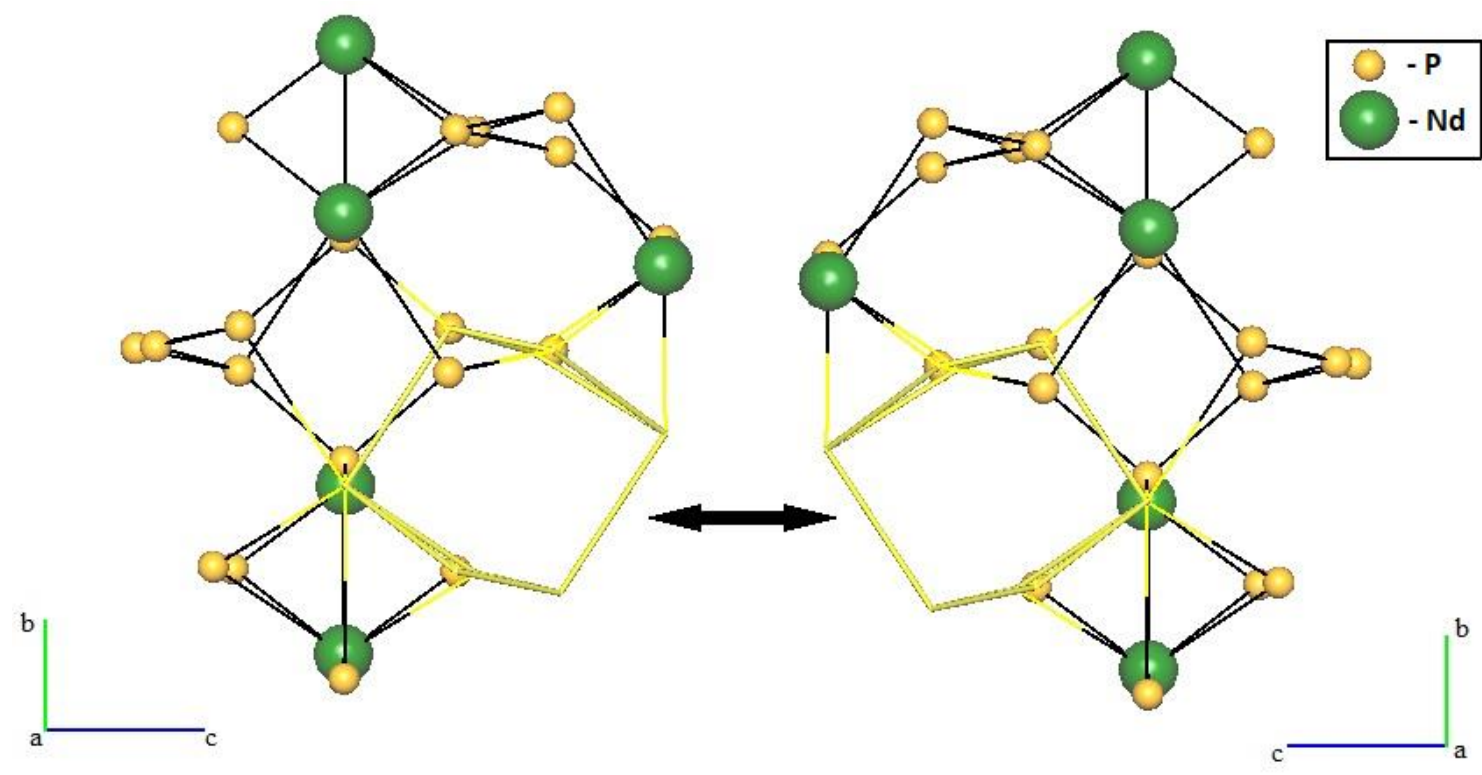

Figure 9. Twinning via a reversal of the c-axis in $\mathrm{NdP}_{5} \mathrm{O}_{14}$. The $8 / 4$ cage is highlighted by the yellow wire frame, showing its position within the cage network. The black arrows show the expected direction of strain, arising from this type of twinning.

While $\mathrm{GdP}_{5} \mathrm{O}_{14}$ manifests the same type and extent of crystallographic twinning seen in $\mathrm{NdP}_{5} \mathrm{O}_{14}$, its 8/4 cage is does not appear to be 'squashed' in the same way. Moreover, there is no report of any mechanical way to induce twinning in $\mathrm{GdP}_{5} \mathrm{O}_{14}$, in stark contrast to $\mathrm{NdP}_{5} \mathrm{O}_{14}$ where twin boundaries may be introduced or removed by applying very low levels of local stress to the crystal; for example, by stroking the crystal with a needle $[3,5]$. The low threshold to strain forming in crystallographic twins of $\mathrm{NdP}_{5} \mathrm{O}_{14}$ is presumably the cause of the 
'squashed' 8/4 cage, in contrast to the case of $\mathrm{GdP}_{5} \mathrm{O}_{14}$ whose $8 / 4$ cage is not similarly squashed and there is no evidence of crystallographic strain. This contrast is presumably a result of the relative strengths of the material frameworks. $\mathrm{GdP}_{5} \mathrm{O}_{14}$ will form a stronger crystal structure than $\mathrm{NdP}_{5} \mathrm{O}_{14}$, since the smaller ionic radius of $\mathrm{Gd}^{3+}$ compared to $\mathrm{Nd}^{3+}$ affords shorter (stronger) $\mathrm{Gd} \cdots \mathrm{O}$ than $\mathrm{Nd} \cdots \mathrm{O}$ coordination bonds, while the electronegativity of gadolinium is also higher than that of neodymium.

\subsection{Phase transitions in $\mathrm{NdP}_{5} \mathrm{O}_{14}$ and $\mathrm{GdP}_{5} \mathrm{O}_{14}$.}

Temperature stability is naturally a key material attribute of a prospective host medium for nuclear waste containment. Since $\mathrm{RP}_{5} \mathrm{O}_{14}$ compounds are known to undergo monoclinic-toorthorhombic second-order phase transitions, it seemed pertinent to track these structural changes as a function of temperature, including the concurrent modulation in the cage volumes of these materials. Moreover, the origin of these phase transitions lies in a variation in crystallographic twinning, whereby the monoclinic angle tends to $90^{\circ}$, whereupon the structure transitions to a non-twinned one with orthorhombic crystal symmetry. Since crystallographic twinning in $\mathrm{NdP}_{5} \mathrm{O}_{14}$ below the transition temperature appears to be the cause of the 'squashed' 8/4 cage through local strain effects, a study that shows how these cage volumes evolve through these phase transitions could also provide additional insights into the anomalous behavior of the $8 / 4$ cage in $\mathrm{NdP}_{5} \mathrm{O}_{14}$.

\subsubsection{Temperature characteristics of the phase transition.}

Previous studies have indicated that the monoclinic-to-orthorhombic transition in $\mathrm{RP}_{5} \mathrm{O}_{14}$ is second order, being gradual at first, but becoming quite pronounced nearer to the transition temperature. The generally accepted transition temperature of $\mathrm{NdP}_{5} \mathrm{O}_{14}$ is $\sim 420 \mathrm{~K}$, while that of $\mathrm{GdP}_{5} \mathrm{O}_{14}$ is $\sim 445 \mathrm{~K}[4-6,10,25]$. The results for $\mathrm{NdP}_{5} \mathrm{O}_{14}$ from our study agree well with those previous reports, as evidenced by the rise in twinning fraction as a function of temperature (Figure 10).

However, the results of our study suggest that the transition for $\mathrm{GdP}_{5} \mathrm{O}_{14}$ occurs at $420-430$ $\mathrm{K}$, rather than at the previously reported 440-450 K. In particular, the twin fraction in the monoclinic structural model suddenly increases from approximately $25 \%$ at $420 \mathrm{~K}$ to approximately $48 \%$ at $430 \mathrm{~K}$ (Figure 10 ), whereupon 6 tends to $90^{\circ}$ at the phase transition. This result is corroborated by the trend observed in the R1 factor for the orthorhombic crystal structure model of $\mathrm{GdP}_{5} \mathrm{O}_{14}$ which surpasses that of the monoclinic model at $420 \mathrm{~K}$ and is maintained at $430 \mathrm{~K}$ and beyond (see Supporting Information).

Further corroboration that the $\mathrm{GdP}_{5} \mathrm{O}_{14}$ transition temperature is indeed in the $420-430 \mathrm{~K}$ range arises from the evaluation of the extinction coefficient of the crystal, as modeled in the monoclinic structural refinements over the full temperature range. From 120-270 K, the extinction coefficient, EXTI, defined according to Sheldrick [14], steadily rises (EXTI $=0.003$ 0.016 ), after which it plateaus at 0.016 ; at $420 \mathrm{~K}$, it sharply decreases, reaching 0.009 at 430 $\mathrm{K}$, before rising again to $\sim 0.015$ by $450 \mathrm{~K}$. Such a temperature-specific drop of the extinction coefficient is indicative of a temporary decrease in crystal quality, which is required for a change of the phase of the crystal structure at this temperature.

Some hysteresis effects in the twinning of the $\mathrm{GdP}_{5} \mathrm{O}_{14}$ crystal were observed upon cooling, reflected in the slightly higher twin fraction observed after returning to low temperatures. As 
the temperature increases from $120 \mathrm{~K}$ to $310 \mathrm{~K}$, the twin fraction averages $~ 5 \%$. However, after transitioning to orthorhombic symmetry and subsequently transitioning back to monoclinic symmetry, the approximate average for the twin fraction as the temperature decreases from $310 \mathrm{~K}$ to $120 \mathrm{~K}$ is $\sim 7 \%$. Unit cell parameters exhibit little or no change, suggesting that any hysteresis is restricted to the twinning fraction. Hysteresis effects for the $\mathrm{NdP}_{5} \mathrm{O}_{14}$ sample were not explored, owing to a weakening of the crystal mount at high temperatures, which rendered the sample unusable after data collection at $480 \mathrm{~K}$.

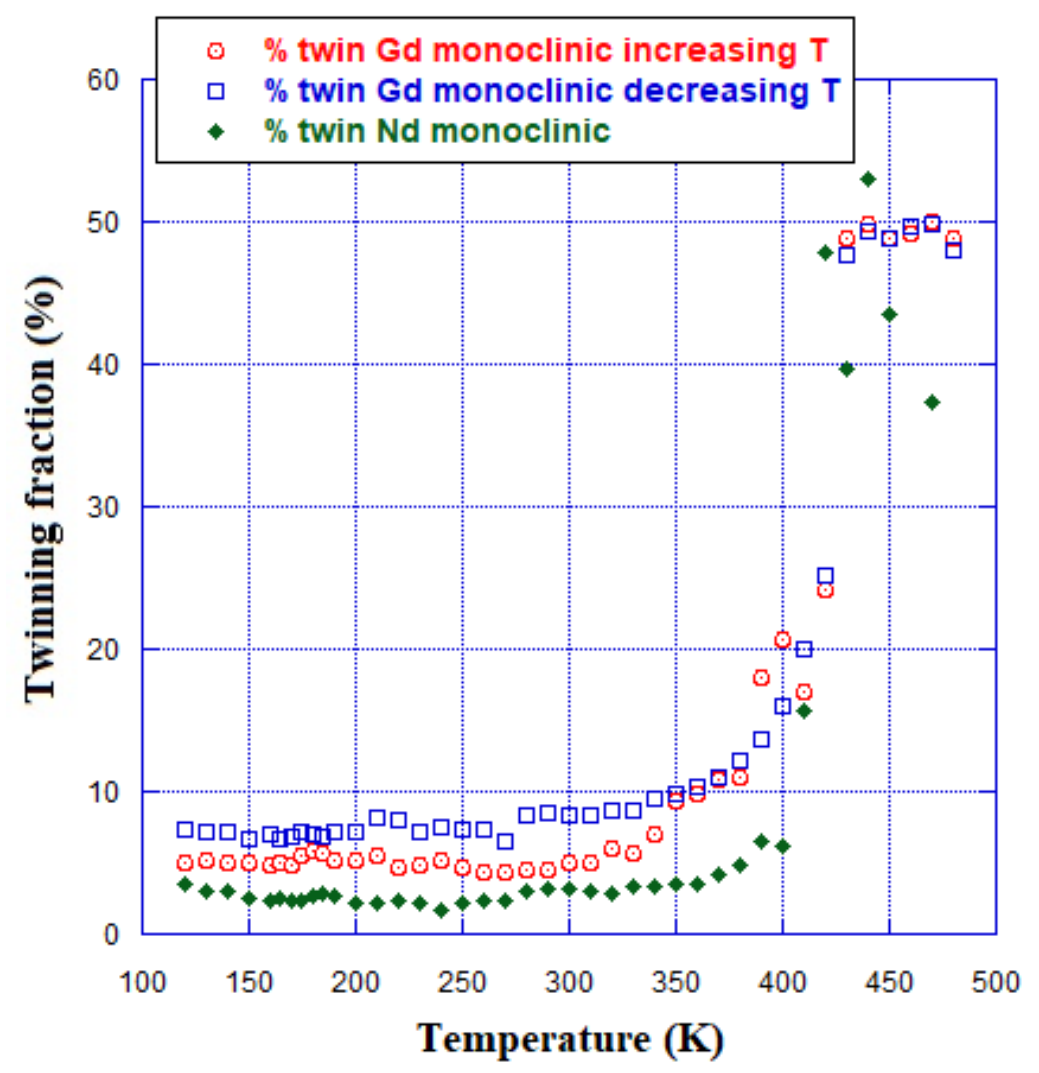

Figure 10. Twinning fractions for $\mathrm{NdP}_{5} \mathrm{O}_{14}$, and $\mathrm{GdP}_{5} \mathrm{O}_{14}$ as a function of temperature, determined from crystal structure refinements of exclusively monoclinic solutions over the full temperature range. Twinning fractions above the transition temperature are therefore artificial since the orthorhombic crystal structures are not twinned, but these artificial values serve as a sensitive parameter by which the phase transition can be tracked.

\subsubsection{Evolution of cage volumes through the phase transitions.}

Comparing temperature-dependent modulations in cage volume between the $\mathrm{NdP}_{5} \mathrm{O}_{14}$ and the $\mathrm{GdP}_{5} \mathrm{O}_{14}$ sample, some notable similarities, and distinct differences become evident. Intuitively, one would expect a fairly steady increase in void space volumes with increasing temperature until the phase transition becomes incipient. This expectation is certainly observed experimentally for the all cages except for 8/4 (Figure 4). The rates of thermal expansion for $\mathrm{NdP}_{5} \mathrm{O}_{14}$ and $\mathrm{GdP}_{5} \mathrm{O}_{14}$ are quite different. Very little thermal expansion is observed in the 10/6 (Figure 6), 16/8 (Figure 7), and 6/4 cages (Figure 8) of $\mathrm{NdP}_{5} \mathrm{O}_{14}$, over the full temperature range studied. Meanwhile, cage volumes of $\mathrm{GdP}_{5} \mathrm{O}_{14}$ increase marginally up 
to $\sim 250 \mathrm{~K}$, beyond which they show greater thermal expansion, with a high degree of fluctuation in view of the incipient phase transition whereby 6 starts to approach $90^{\circ}$.

Regarding the $8 / 4$ cage, its thermal expansion in $\mathrm{NdP}_{5} \mathrm{O}_{14}$ seems to increase essentially monotonically until about $400 \mathrm{~K}$. In contrast, the 8/4 cage in $\mathrm{GdP}_{5} \mathrm{O}_{14}$ appears to start contracting with increasing temperature beyond about $300 \mathrm{~K}$ until it approaches the phase transition, whereupon its volume fluctuates substantially; its original volume is recovered upon lowering the temperature back below $300 \mathrm{~K}$ but only via a significant level of thermal hysteresis (Figure 4). This level of thermal contraction in GdP5O14 is such that it tends towards the volume of its 'squashed' $\mathrm{NdP}_{5} \mathrm{O}_{14}$ analogue in the region 350-450 K. While it was discussed above that the stronger nature of the $\mathrm{GdP}_{5} \mathrm{O}_{14}$ lattice may allow it to resist the squashed nature of its $\mathrm{NdP}_{5} \mathrm{O}_{14}$ counterpart at low temperatures, these anomalous thermal effects observed above room temperature may suggest that it cannot overcome them at elevated temperatures. This thermal instability suggests a limit to its use in nuclear waste storage, should this result in radiation leakage of an encapsulated ion from this particular cage in $\mathrm{GdP}_{5} \mathrm{O}_{14}$. Meanwhile, the squashed nature of the 8/4 cage in $\mathrm{NdP}_{5} \mathrm{O}_{14}$ indicates that containment of nuclear waste material within the 8/4 cage of $\mathrm{RP}_{5} \mathrm{O}_{14}$ framework materials would be inadvisable. Fortunately, in the aforementioned recent screening study of volumes of empty cages in 628 phosphate framework structures to pinpoint potential hosts for encapsulating guest materials such as the nuclear waste, no 8-nodal cages in any of these possible phosphate structures were of the right size to accommodate uranium, plutonium or caesium ions as guests. It would therefore appear that encapsulation of spent nuclear waste within 8/4 cages of phosphate frameworks is unlikely in any case.

Notwithstanding this structural anomaly in the 8/4 cage, $\mathrm{GdP}_{5} \mathrm{O}_{14}$ and $\mathrm{NdP}_{5} \mathrm{O}_{14}$ framework materials would seem pretty rigid over a wide temperature range, including the elevated temperatures that are associated with spent fuels that are cooling within nuclear waste streams. They would therefore seem able to encapsulate nuclear waste materials without leakage.

\section{CONCLUSIONS}

$\mathrm{NdP}_{5} \mathrm{O}_{14}$ and $\mathrm{GdP}_{5} \mathrm{O}_{14}$ have been assessed for their prospects as host media to encapsulate guests, such as $\mathrm{UO}_{2}$ or $\mathrm{PuO}_{2}$, for nuclear waste containment. To this end, the crystal structures of these nanoporous host frameworks were determined, whereupon the topological characteristics of each type of nanoporous cage was classified, and the associated cage volume was calculated. One of these void spaces, classified as an 8/4 cage, appears to be 'squashed' within the structure of $\mathrm{NdP}_{5} \mathrm{O}_{14}$ but curiously not in $\mathrm{GdP}_{5} \mathrm{O}_{14}$ at low temperatures, even though the two compounds are otherwise isostructural. Nonetheless, the 8/4 cage in $\mathrm{GdP}_{5} \mathrm{O}_{14}$ was found to contract at elevated temperatures, seemingly overcoming the stronger nature of the $\mathrm{GdP}_{5} \mathrm{O}_{14}$ lattice, compared with that of $\mathrm{NdP}_{5} \mathrm{O}_{14}$, that presumably withstands contraction forces at low temperatures. A topological analysis revealed that the 8/4 cage is much weaker than the other types of cages in $\mathrm{RP}_{5} \mathrm{O}_{14}$ frameworks, because its structural scaffold contains few cross-links that would otherwise give it more strength. Crystallographic twinning in $\mathrm{NdP}_{5} \mathrm{O}_{14}$, which exposes this $8 / 4$ cage to local strain, also makes its weak character particularly susceptible to local strain effects, to an extent that twinning in $\mathrm{NdP}_{5} \mathrm{O}_{14}$ can be induced by a small mechanical force, such as a crystal of $\mathrm{NdP}_{5} \mathrm{O}_{14}$ being stroked by a needle $[3,5]$. Multi-temperature single-crystal $X$-ray diffraction studies of $\mathrm{GdP}_{5} \mathrm{O}_{14}$ and $\mathrm{NdP}_{5} \mathrm{O}_{14}(\mathrm{~T}=$ 120-480 K) showed how the strength of the 8/4 cage in $\mathrm{GdP}_{5} \mathrm{O}_{14}$ starts to falter above $300 \mathrm{~K}$, 
leading to thermal contraction with increasing temperature such that it tends toward the volume of the 8/4 cage in $\mathrm{NdP}_{5} \mathrm{O}_{14}$ at elevated temperatures. The other types of cages in these $\mathrm{RP}_{5} \mathrm{O}_{14}$ structures exhibited fairly regular behavior in thermal expansion, until the temperature approached the monoclinic-to-orthorhombic second-order phase transitions of $\mathrm{NdP}_{5} \mathrm{O}_{14}$, and $\mathrm{GdP}_{5} \mathrm{O}_{14}$, identified at $\mathrm{T} \sim 420 \mathrm{~K}$, and 420-430 $\mathrm{K}$, respectively. The multitemperature studies also enabled the thermal stability of these host frameworks to be assessed in terms of their applicability to the environmental conditions that are typical in processing and storing nuclear waste. Both $\mathrm{NdP}_{5} \mathrm{O}_{14}$ and $\mathrm{GdP}_{5} \mathrm{O}_{14}$ compounds appear to present good prospects as host media for nuclear waste containment pending that the 8/4 cage is not involved in guest encapsulation.

Although it is beyond the remit of this study, further investigations into the suitability of rareearth ultraphosphates for the purposes of nuclear waste storage will also need to take several other factors into account. In particular, physical and chemical considerations from daughter products resulting from continued radioactive decay must be investigated, as well as the effects of extensive radiation exposure on the stability of the host structure, including dose, type of radiation, and decay energies. These additional factors are not trivial, requiring considerable testing and thought, given that the composition of the anticipated radionuclides to be included in the host phosphate material will most likely vary significantly from waste stream to waste stream [1].

\section{ACKNOWLEDGEMENTS}

J.M.C. thanks the 1851 Royal Commission of the Great Exhibition for the 2014 Fellowship in Design, hosted by Argonne National Laboratory where work done was supported by DOE Office of Science, Office of Basic Energy Sciences, under contract No. DE-AC02-06CH11357.

\section{SUPPORTING INFORMATION}

Supporting Information available: Crystallographic Information Files (CIFs) for $\mathrm{NdP}_{5} \mathrm{O}_{14}$ and $\mathrm{GdP}_{5} \mathrm{O}_{14}$ as a function of temperature $(\mathrm{T}=120-480 \mathrm{~K})$ showing both monoclinic and orthorhombic crystal structure refinements in the region of the second-order monoclinic-toorthorhombic phase transition. $\mathrm{CIFs}$ for $\mathrm{GdP}_{5} \mathrm{O}_{14}$ are given for studies on increasing and decreasing temperature, demonstrating its thermal hysteresis above $\mathrm{T}=300 \mathrm{~K}$.

\section{CONFLICTS OF INTEREST}

The authors declare the absence of any potentially competing financial interests.

\section{REFERENCES}

[1] A. J. Cramer, J. M. Cole, Removal or Storage of Environmental Pollutants and Alternative Fuel Sources with Inorganic Adsorbents via Host-Guest Encapsulation, J. Mater. Chem. A, 2017, 5, 10746.

[2] A. J. Cramer, J. M. Cole, Topological Analysis of Void Space in Phosphate Frameworks: Assessing Storage Properties for the Environmentally Important Guest Molecules and lons: $\mathrm{CO}_{2}, \mathrm{H}_{2} \mathrm{O}, \mathrm{UO}_{2}, \mathrm{PuO}_{2}, \mathrm{U}, \mathrm{Pu}, \mathrm{Sr}^{2+}, \mathrm{Cs}^{+}, \mathrm{CH}_{4}$, and $\mathrm{H}_{2}, \mathrm{ACS}$ Sustain. Chem. Eng., 2016, 4, 4094. 
[3] H. P. Weber, B. C. Tofield, P. F. Liao, Ferroelastic Behavior and the Monoclinic-toOrthorhombic Phase Transition in $\mathrm{MP}_{5} \mathrm{O}_{14}$ (M = La-Tb), Phys. Rev. B, 1975, 11, 1152.

[4] T. Kobayashi, T. Sawada, H. Ikeo, K. Muto, J. Kai, Ferroelasticity in $\mathrm{ReP}_{5} \mathrm{O}_{14}$ (Re: LaTb), J. Phys. Soc. Japan, 1976, 40, 595.

[5] J. P. Budin, A. Milatos-Roufos, N. Duc Chinh, G. Le Roux, Ferroelastic Behavior of $\mathrm{Nd}_{x} \mathrm{La}_{1-\mathrm{x}} \mathrm{P}_{5} \mathrm{O}_{14}$ Crystals, J. Appl. Phys., 1975, 46, 2867.

[6] Q. R. Cai, G. X. Lan, H. F. Wang, G. Y. Hong, The Effect of Pressure on the Ferroelastic Phase Transition in $\mathrm{LnP}_{5} \mathrm{O}_{14}$, J. Phys. Chem. Solids, 1990, 51, 279.

[7] G. Errandonea, J. Sapriel, Polarized Raman Study of the Ferroelastic Transition in Lanthanum Pentaphosphate, Solid State Commun., 1979, 32, 391.

[8] G. M. Loiacono, M. Delfino, W. A. Smith, Specific Heats of $\mathrm{NdP}_{5} \mathrm{O}_{14}$ and $\mathrm{PrP}_{5} \mathrm{O}_{14}$ near their Ferroelastic Phase Transitions, Appl. Phys. Lett., 1978, 32, 595.

[9] I. Takei, T. Kobayashi, S. Nakamura, K. Yamana, Atomic Force Microscopy Observation of Ferroelastic Domain Boundary in $\mathrm{GdP}_{5} \mathrm{O}_{14}$, J. Phys. Soc. Japan, 1994, 63, 2477.

[10] X. R. Huang, Z. W. Hu, S. S. Jiang, J. H. Jiang, Y. L. Tian, Y. Han, J. Y. Wang, Synchrotron Radiation Topography Studies of Twinning in $\mathrm{NdP}_{5} \mathrm{O}_{14}$ Crystals, J. Appl. Phys., 1994, 75, 7756.

[11] H. G. Danielmeyer, J. P. Jeser, E. Schönherr, W. Stetter, The Growth of Laser Quality $\mathrm{NdP}_{5} \mathrm{O}_{14}$ Crystals, J. Cryst. Growth, 1974, 22, 298.

[12] Rigaku. CrystalClear-SM Expert and CrystalStructure. Rigaku: The Woodlands, Texas, USA 2009.

[13] T. Higashi, ABSCOR. Rigaku Corporation: Tokyo, Japan 1995.

[14] G. M. Sheldrick, A Short History of SHELX, Acta Crystallogr. A, 2008, 64, 112.

[15] V. A. Blatov, Nanocluster Analysis of Intermetallic Structures with the Program Package TOPOS, Struct. Chem., 2012, 23, 955.

[16] O. Delgado-Friedrichs, M. O'Keeffe, Crystal Nets as Graphs: Terminology and Definitions, J. Solid State Chem., 2005, 178, 2480.

[17] C. Bonneau, O. Delgado-Friedrichs, M. O'Keeffe, O. Yaghi, Three-Periodic Nets and Tilings: Minimal Nets, Acta Crystallogr. A., 2004, 60, 517.

[18] V. A. Blatov, O. Delgado-Friedrichs, M. O'Keeffe, D. M. Proserpio, Three-Periodic Nets and Tilings: Natural Tilings for Nets, Acta Crystallogr. A., 2007, 63, 418.

[19] K.-R. Albrand, R. Attig, J. Fenner, J. P. Jeser, D. Mootz, Crystal Structure of the Laser Material $\mathrm{NdP}_{5} \mathrm{O}_{14}$, Mater. Res. Bull., 1974, 9, 129.

[20] J. M. Cole, M. R. Lees, J. A. K. Howard, R. J. Newport, G. A. Saunders, E. Schönherr, Crystal Structures and Magnetic Properties of Rare-Earth Ultraphosphates, $\mathrm{RP}_{5} \mathrm{O}_{14}(\mathrm{R}$ = La, Nd, Sm, Eu, Gd), J. Solid State Chem., 2000, 150, 377.

[21] H. Ettis, H. Naïli, T. Mhiri, Reinvestigation of the $\mathrm{GdP}_{5} \mathrm{O}_{14}$ Crystal Structure at Room Temperature and Magnetic Properties, Mater. Chem. Phys., 2007, 102, 275.

[22] H. Y. P. Hong, Crystal Structures of Neodymium Metaphosphate $\left(\mathrm{NdP}_{3} \mathrm{O}_{9}\right)$ and ultraphosphate $\left(\mathrm{NdP}_{5} \mathrm{O}_{14}\right)$, Acta Crystallogr. Sect. B Struct. Crystallogr. Cryst. Chem., 1974, 30, 468.

[23] Y.-H. Lin, N.-H. Hu, Q.-G. Zhou, S.-X. Wu, Crystal Structure of Gadolinium Pentaphosphate, Yingyong Huaxue, 1983, 1, 33.

[24] H. Schulz, K.-H. Thiemann, J. Fenner, The High-Temperature Phase of $\mathrm{NdP}_{5} \mathrm{O}_{14}$, Mater. Res. Bull., 1974, 9, 1525. 
[25] S. Ashida, Y. Ito, Optical Properties and Phase Transition in Neodymium Pentaphosphate $\left(\mathrm{NdP}_{5} \mathrm{O}_{14}\right)$ Single Crystals, Mineral. J., 1976, 8, 215.

GRAPHICAL ABSTRACT

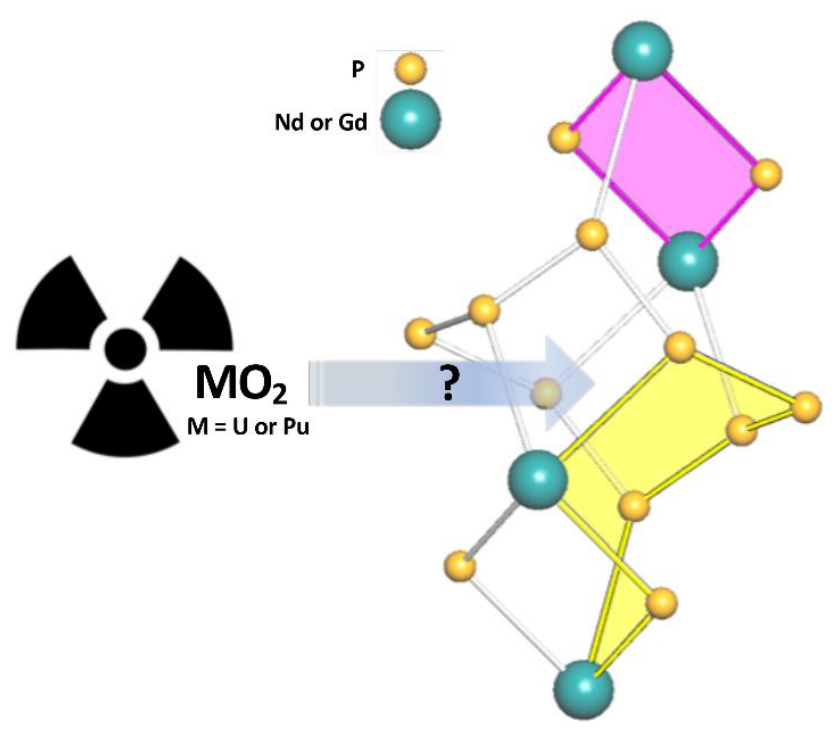

\section{HIGHLIGHTS}

- The temperature-dependent cage topologies of $\mathrm{RP}_{5} \mathrm{O}_{14}(\mathrm{R}=\mathrm{Nd}$ or $\mathrm{Gd})$ were examined

- A topological analysis revealed similar cage structure for $\mathrm{RP}_{5} \mathrm{O}_{14}(\mathrm{R}=\mathrm{Nd}$ or $\mathrm{Gd})$

- One exception: the 8/4 cage topology of $\mathrm{NdP}_{5} \mathrm{O}_{14}$ lacks structural cross-linking

- Volume $\left(\mathrm{UO}_{2}\right.$ or $\left.\mathrm{PuO}_{2}\right) \gg 8 / 4$ topology in $\mathrm{RP}_{5} \mathrm{O}_{14}(\mathrm{R}=\mathrm{Nd}$ or $\mathrm{Gd})$

- $\mathrm{RP}_{5} \mathrm{O}_{14}(\mathrm{R}=\mathrm{Nd}$ or $\mathrm{Gd})$ are good prospective host media for nuclear waste storage 